\title{
NONNEGATIVE EIGEN FUNCTIONS OF LAPLACE-BELTRAMI OPERATORS ON SYMMETRIC SPACES
}

\author{
BY R. G. LAHA ${ }^{1}$
}

Communicated by G. D. Mostow, August 25, 1967

1. Introduction. Let $G$ be a connected semisimple Lie group with a finite center and let $K$ be a maximal compact subgroup of $G$ and let $X=G / K$ be the homogeneous space of left cosets $g K(g \in G)$ of the group $G$ with respect to the subgroup $K$. Then it is known that a $G$-invariant Riemannian metric can be introduced in the space $X$ so that $X$ becomes a Riemannian symmetric space of nonpositive curvature. Let $\$$ be the Lie algebra of $G$ and let $\Omega$ be the subalgebra of $\$ 3$ corresponding to the subgroup $K$. Let $\mathfrak{B}$ be the orthogonal complement of $\Omega$ in $\mathbb{S}$ with respect to the Killing form $\langle$,$\rangle of the alge-$ bra $\mathbb{S}$. Let $\mathfrak{A}$ be a maximal abelian subspace of $\mathfrak{B}$. Then $\mathfrak{A}$ is a Cartan subalgebra of the symmetric space $X$. Let $\mathfrak{X}^{\prime}$ be the set of all regular elements in $\mathfrak{A}$ and let $\mathfrak{X}^{+}$be a fixed component (connected) in $\mathfrak{X}^{\prime}$. Then the set $\mathfrak{A}^{+}$is a Weyl chamber in the space $\mathfrak{A}$. Let $\mathfrak{A}^{*}$ be the dual space of the space $\mathfrak{A}$. Then the space $\mathfrak{A}^{*}$ can be identified with the space $\mathfrak{A}$ by means of the Killing form $\langle$,$\rangle of B$ in the usual manner. Let $\alpha \in \mathfrak{I}^{*}$. We set

$$
\mathfrak{S}_{\alpha}=\{X \in \mathbb{S}:[H, X]=\alpha(H) X \text { for all } H \in \mathfrak{A}\},
$$

$d_{\alpha}=\operatorname{dim}\left(\xi_{\alpha}\right.$. Then $\alpha$ is said to be a root of the space $X$ with respect to the Cartan subalgebra $\mathfrak{A}$, if $d_{\alpha}>0$. A root $\alpha$ is said to be positive if $\alpha(H)>0$ for all $H \in \mathfrak{Q}^{+}$. Let $P$ be the set of all positive roots of $X$ with respect to $\mathfrak{A}$. We set

$$
\begin{aligned}
\rho=\frac{1}{2} \sum_{\alpha \in P} d_{\alpha} \alpha ; & \mathfrak{N}=\sum_{\alpha \in P} \mathfrak{H}_{\alpha} \\
A=\exp (\mathfrak{R}) ; & N=\exp (\mathfrak{R}) .
\end{aligned}
$$

Then we have the Iwasawa decomposition: $G=K A N$ where $A$ and $N$ are connected commutative and nilpotent subgroups of $G$ respectively.

Let $a \in A$. Then there exists a unique element $H \in \mathfrak{P}$ such that $a=\exp H$. We then write $H=\ln a$.

2. Some basic prerequisites. We now give some results from [4]

1 This work was supported by the National Science Foundation through grant NSF-GP-6175. 
which are instrumental for the formulation of the main theorems in the next section.

A horocycle $\xi$ in $X$ is an orbit in the space $X$ of a group of the form $g N g^{-1}, g \in G$. Let $\Xi$ be the set of all horocycles in $X$. Let $M$ be the centralizer of $A$ in $K$. Then the set $\Xi$ can be identified with the homogeneous space $G / M N$. Moreover the homogeneous space $K / M$ can be identified with the set of all Weyl chambers contained in all maximal abelian subspaces of the space $\mathfrak{B}$.

Let $x_{0}=\{K\}$ be the origin in $X$ and let $\xi_{0}=N \cdot x_{0}$ be the origin in $\Xi$. Let $\xi \in \Xi$ be arbitrary. Then the horocycle $\xi$ can be written as

$$
\xi=k a \xi_{0}
$$

where $a \in A$ is unique and $k \in K$ is unique $(\bmod M)$. Here the Weyl chamber $k M$ is said to be normal to the horocycle $\xi$ and the element $a \in A$ is said to be the complex distance from $x_{0}$ to $\xi$. We set $B=K / M$. There the space $B$ can be identified with the Furstenberg boundary $B(G)$ (cf. [2]).

Let $x \in X, b \in B$. Let $\xi(x, b)$ be the horocycle with normal $b$ and passing through the point $x$. Let $a(x, b)$ be the complex distance from $x_{0}$ to $\xi(x, b)$. Let $H(x, b)=\ln a(x, b)$ so that $H(x, b) \in \mathfrak{A}$. It is shown in [4] that the elements $H(x, b)$ play an essential role in defining the spherical Fourier transform of an arbitrary function $f \in C_{c}^{\infty}(X)$.

3. Main results. Let $\Delta$ be the Laplace-Beltramioperator in the space $X$ and let $c$ be some real number. We first give a complete description of the cone of all nonnegative solutions of the equation

$$
\Delta f=c f .
$$

It is shown in [5] that, for $c<-\langle\rho, \rho\rangle$, the equation (1) does not have a nonnegative solution (except the trivial solution $f=0$ ). Hence we consider only the case $c \geqq-\langle\rho, \rho\rangle$.

A nonnegative solution $f$ of $(1)$ is said to be normalized if $f\left(x_{0}\right)=1$. A nonnegative solution $f$ of (1) is said to be minimal, if every nonnegative solution of (1) which does not exceed $f$ is a constant multiple of $f$.

Let $c \geqq-\langle\rho, \rho\rangle$. We set

$$
\mathfrak{A}_{c}=\{H \in \mathfrak{N}:\langle H, H\rangle=c+\langle\rho, \rho\rangle\} \quad \text { and } \quad \mathfrak{A}_{c}^{+}=\mathfrak{A}^{+} \cap \mathfrak{A}_{c} \text {. }
$$

Let $b \in B, \lambda \in \mathfrak{U}_{c}^{+}$. We now define the function $\phi_{b, \lambda}$ on $X$ by the formula

$$
\phi_{b, \lambda}(x)=e^{(\lambda+p)(H(x, b))} \quad(x \in X) .
$$


THEOREM 1. The set of all normalized minimal solutions of the equation (1) coincide with the set of all functions

$$
\left\{\phi_{b, \lambda}: b \in B, \lambda \in \mathfrak{A}_{c}^{+}\right\} .
$$

The proof can be carried out by using the method of induction on the rank of the symmetric space $X$ as in [5].

Theorem 2. A function $f$ is a nonnegative solution of (1) if and only if $f$ can be represented in the form

$$
f(x)=\int_{B \times \mathfrak{u}_{c}} \phi_{b, \lambda}(x) d \mu(b, \lambda) .
$$

Here $\mu$ is a finite positive Radon measure on $B \times \mathfrak{A}_{c}^{+}$which is uniquely determined by $f$.

The proof is an immediate consequence of Theorem 1 and Choquet's Theorem (cf. [1]).

Let $\mathfrak{D}(X)$ be the algebra of all $G$-invariant differential operators on $X$. Then a function $f \in C^{\infty}(X)$ is said to be semi-spherical, if $f$ is an eigen function of every differential operator $D \in \mathfrak{D}(X)$ and moreover satisfies the relation $f\left(x_{0}\right)=1$. Clearly a semi-spherical function $f$ is a spherical function on $X$ if and only if $f$ is $K$-invariant.

TheOREM 3. A function $f$ on $X$ is a nonnegative semi-spherical function on $X$ if and only if $f$ can be represented in the form

$$
f(x)=\int_{B} \phi_{b, \lambda}(x) d \mu(b) .
$$

Here $\lambda \in \mathfrak{Y}^{+}$and $\mu$ is a finite positive Radon measure on $B$ such that $\int_{B} d \mu(b)=1$. Moreover the pair $(\mu, \lambda)$ is determined uniquely by the function $f$.

THEOREM 4. A function $f$ on $X$ is a nonnegative spherical function on $X$ if and only if $f$ can be represented in the form

$$
f(x)=\int_{B} \phi_{b, \lambda}(x) d b .
$$

Here $\lambda \in \mathfrak{A}^{+}$and $d b$ is the unique $K$-invariant positive measure on $B$ such that $\int_{B} d b=1$.

REMARK 1. This theorem can be considered as a special case of a more general result of Harish-Chandra [3] which gives the integral representation of an arbitrary complex-valued spherical function on $X$. 
A function $f$ on $X$ is said to be harmonic, if $f$ satisfies the equation $\Delta f=0$.

THEOREM 5. A function $f$ on $X$ is a bounded nonnegative harmonic function on $X$ if and only if $f$ can be represented in the form

$$
f(x)=\int_{B} e^{2 \rho(H(x, b))} \hat{f}(b) d b .
$$

Here $\hat{f}$ is a bounded nonnegative measurable function on $B$ and is determined uniquely by $f$ almost everywhere on $B$.

Remark 2. Here the function $e^{2 \rho(H(x, b))}$ is the Poisson kernel and the formula (6) is the analog of Poisson integral formula for bounded nonnegative harmonic functions on symmetric spaces (cf. [2]).

\section{REFERENCES}

1. G. Choquet, Existence et unicité des représentations intégrales au moyen des points extrémaux dans les cônes convexes, Seminaire Bourbaki, Exp. 139, (1956); C. R. Acad. Sci. Paris 203 (1956), 699-702.

2. H. Furstenberg, A Poisson formula for semi-simple Lie groups, Ann. of Math. (2) 77 (1963), 335-386.

3. Harish-Chandra, Spherical functions on a semi-simple Lie group. I, Amer. J. Math. 80 (1958), 241-310.

4. S. Helgason, $A$ duality in integral geometry on symmetric spaces, Proc. U. S.Japan Seminar in Differential Geometry, Kyoto, 1965.

5. F. I. Karpelevich, Geometry of geodesics and eigen functions of Laplace-Beltrami operators on symmetric spaces, Trudy Moskov. Mat. Obšc. 14 (1965), 48-185. (Russian)

The Catholic University of America 